\title{
GENERALIZED BORN-JORDAN DISTRIBUTIONS AND APPLICATIONS
}

\author{
ELENA CORDERO, MAURICE DE GOSSON, MONIKA DÖRFLER, AND FABIO NICOLA
}

\begin{abstract}
The quadratic nature of the Wigner distribution causes the appearance of unwanted interferences. This is the reason why engineers, mathematicians and physicists look for related time-frequency distributions, many of them are members of the Cohen class [7. Among them, the Born-Jordan distribution has recently attracted the attention of many authors, since the so-called "ghost frequencies "are damped quite well, and the noise is in general reduced. The very insight relies on the kernel of such a distribution, which contains the sinus cardinalis sinc, which can be viewed as the Fourier transform of the first B-Spline $B_{1}$. Replacing the function $B_{1}$ with the spline or order $n$, denoted by $B_{n}$, on the Fourier side we obtain $(\operatorname{sinc})^{n}$, whose decay at infinity increases with $n$. We introduce the Cohen's Kernel $\Theta^{n}\left(z_{1}, z_{2}\right)=\operatorname{sinc}^{n}\left(z_{1} \cdot z_{2}\right)$ and study the properties of the related time-frequency distribution $Q^{n}$, which we call generalized Born-Jordan distribution.
\end{abstract}

\section{INTRODUCTION}

The study of signals in the time-frequency plane is a subject which involves many different people: engineers, physicists, mathematicians, both on theoretical and applied levels, cf.,e.g., [6, 7, 24, 33].

One of the most popular time-frequency representation of a signal $f$ is the Wigner distribution

$$
W f(x, \omega)=\int_{\mathbb{R}^{d}} f\left(x+\frac{y}{2}\right) \overline{f\left(x-\frac{y}{2}\right)} e^{-2 \pi i y \omega} d y, \quad x, \omega \in \mathbb{R}^{d},
$$

where the signal $f$ can be thought as a function in $L^{2}\left(\mathbb{R}^{d}\right)$ or more generally as a tempered distribution $\left(f \in \mathcal{S}^{\prime}\left(\mathbb{R}^{d}\right)\right)$.

It is well known that the quadratic nature of this representation causes the appearance of interferences between several components of the signal. To overcome this issue, the so-called Cohen class was introduced in [6] and widely studied by many authors (see [2] and references therein). We address the interested reader to the textbooks [7, 33].

2010 Mathematics Subject Classification. Primary 42B10, Secondary 42B37.

Key words and phrases. Time-frequency analysis, Wigner distribution, Born-Jordan distribution, B-Splines, Interferences, wave-front set, modulation spaces, Fourier Lebesgue spaces. 
A member of the Cohen class $Q f$ is obtained by convolving the Wigner representation $W f$ with a distribution $\theta \in \mathcal{S}^{\prime}\left(\mathbb{R}^{2 n}\right)$

$$
Q f=W f * \theta .
$$

A possible choice is $\theta=\mathcal{F}_{\sigma} \Theta^{1}$, with $\mathcal{F}_{\theta} \Theta^{1}$ being the symplectic Fourier transform of the Cohen kernel

$$
\Theta^{1}(x, \omega)=\operatorname{sinc}(x \omega)= \begin{cases}\frac{\sin (\pi x \omega)}{\pi x \omega} & \text { for } x \omega \neq 0 \\ 1 & \text { for } x \omega=0\end{cases}
$$

$\left(x \omega=x \cdot \omega\right.$ denoting the scalar product in $\left.\mathbb{R}^{d}\right)$. In this way we obtain the BornJordan distribution:

$$
Q^{1} f=W f * \mathcal{F}_{\sigma}\left(\Theta^{1}\right), \quad f \in L^{2}\left(\mathbb{R}^{d}\right),
$$

see [2, 6, 7, 8, 10, 26, 29, 33] and the references therein.

We can create other interesting kernels and related distributions using the Bspline functions $B_{n}$. Recall that the sequence of B-splines $\left\{B_{n}\right\}_{n \in \mathbb{N}_{+}}$, is defined inductively as follows. The first B-Spline is

$$
B_{1}(t)=\chi_{\left[-\frac{1}{2}, \frac{1}{2}\right]}(t)
$$

whereas, assuming that we have defined $B_{n}$, for some $n \in \mathbb{N}_{+}$, the spline $B_{n+1}$ is defined by

$$
B_{n+1}(t)=\left(B_{n} * B_{1}\right)(t)=\int_{\mathbb{R}} B_{n}(t-y) B_{1}(y) d y=\int_{-\frac{1}{2}}^{\frac{1}{2}} B_{n}(t-y) d y .
$$

The spline $B_{n}$ is a piecewise polynomial of degree at most $n-1, n \in \mathbb{N}_{+}$, and satisfying $B_{n} \in \mathcal{C}^{n-2}(\mathbb{R}), n \geq 2$. For the main properties we refer, e.g., to [4].

Observe that $\operatorname{sinc}(\xi)=\mathcal{F} B_{1}(\xi)$ and by induction we infer

$$
\operatorname{sinc}^{n}(\xi)=\mathcal{F} B_{n}(\xi), \quad n \in \mathbb{N}_{+} .
$$

Definition 1.1. For $n \in \mathbb{N}$, the nth Born-Jordan kernel is the function on $\mathbb{R}^{2 d}$ defined by

$$
\Theta^{n}(x, \omega)=\operatorname{sinc}^{n}(x \omega), \quad(x, \omega) \in \mathbb{R}^{2 d} .
$$

The Born-Jordan distribution of order $n(B J D n)$ is given by

$$
Q^{n} f=W f * \mathcal{F}_{\sigma}\left(\Theta^{n}\right), \quad f \in L^{2}\left(\mathbb{R}^{d}\right) .
$$

The cross-BJDn is given by

$$
Q^{n}(f, g)=W(f, g) * \mathcal{F}_{\sigma}\left(\Theta^{n}\right), \quad f, g \in L^{2}\left(\mathbb{R}^{d}\right) .
$$

We write $Q^{n}(f, f)=Q^{n} f$, for every $f \in L^{2}\left(\mathbb{R}^{d}\right)$.

For $n=0, \Theta^{0} \equiv 1$ and $\mathcal{F}_{\sigma}(1)=\delta$, so that $Q^{0} f=W f$, the Wigner distribution of the signal $f$. 
Roughly speaking, the class of the BJDn's form a subclass of the Cohen class, containing the Wigner distribution. This subclass will play an important role in the applications, since its members display a great capacity of damping interferences and such reduction increases with $n$.

In this paper we show the different facets of this phenomenon, from visual comparisons to rigorous mathematical explanations. Motivated by this issue, a thorough study of such distributions and related pseudodifferential calculus is performed. In this way we show the many connections and uses of the BJDn's $Q^{n}$, paving the way to possible other interesting and useful applications.

The main subjects treated in this paper are the following:

(i) Regularity and Smoothness Properties of $Q^{n}$;

(ii) Damping of interferences in comparison with the Wigner distribution;

(iii) Visual comparison in dimension $d=1$ between $Q^{n}$ and the Wigner Distribution;

(iv) Born-Jordan quantization of order $n$ and related pseudodifferential calculus.

The most suitable framework to handle these aspects can be found in the scale of modulation spaces (see [18] and also the textbook [30]), recalled in Subsection 2.3. In short, we first introduce another time-frequency representation: the short-time Fourier transform (STFT). Fix a Schwartz function $g \in \mathcal{S}\left(\mathbb{R}^{d}\right) \backslash\{0\}$ (the so-called window). We define the short-time Fourier transform of $f$ as

$$
V_{g} f(x, \omega)=\int_{\mathbb{R}^{d}} f(y) \overline{g(y-x)} e^{-2 \pi i y \omega} d y, \quad(x, \omega) \in \mathbb{R}^{2 d} .
$$

For $1 \leq p, q \leq \infty$, the (unweighted) modulation space $M^{p, q}\left(\mathbb{R}^{d}\right)$ is the subspace of tempered distributions $f$ such that

$$
\|f\|_{M^{p, q}}:=\left(\int_{\mathbb{R}^{d}}\left(\int_{\mathbb{R}^{d}}\left|V_{g} f(x, \omega)\right|^{p} d x\right)^{q / p} d \omega\right)^{1 / q}<\infty
$$

(with obvious modifications for $p=\infty$ or $q=\infty$ ). Roughly speaking, a signal $f$ is in $M^{p, q}\left(\mathbb{R}^{d}\right)$ if it decays at infinity as a function in $\ell^{p}\left(\mathbb{Z}^{d}\right)$ whereas displays a smoothness measured in the scale $\mathcal{F} L^{q}\left(\mathbb{R}^{d}\right)$.

Their images under the Fourier transform are the modulation spaces $W\left(\mathcal{F} L^{p}, L^{q}\right)\left(\mathbb{R}^{d}\right)$ (also known as Wiener amalgam spaces, see subsection 2.3 below). Observe that a tempered distribution $f$ in $W\left(\mathcal{F} L^{p}, L^{q}\right)\left(\mathbb{R}^{d}\right)$ decays at infinity as a function in $\ell^{q}\left(\mathbb{Z}^{d}\right)$ whereas locally behaves as a function in $\mathcal{F} L^{p}\left(\mathbb{R}^{d}\right)$. Of particular interest is the space $W\left(\mathcal{F} L^{1}, L^{\infty}\right)\left(\mathbb{R}^{d}\right)$, which is an algebra under pointwise multiplication.

With all these instruments at hand, we can exhibit the results obtained in this paper.

Regularity of $Q^{n}$. It is intuitively clear that the Born-Jordan distribution of order $n$ of a signal, as for the classical one $Q^{1}$, is certainly not rougher than the 
corresponding Wigner distribution. We shall show in Proposition 4.2 that the nth-Cohen kernel belongs to the Wiener amalgam space $W\left(\mathcal{F} L^{1}, L^{\infty}\right)$, for every $n \in \mathbb{N}_{+}$. This is the key tool for proving the following result (cf. Theorem 5.1):

Theorem 1.2. Let $f \in \mathcal{S}^{\prime}\left(\mathbb{R}^{d}\right)$ be a signal, with $W f \in M^{p, q}\left(\mathbb{R}^{2 d}\right)$ for some $1 \leq$ $p, q \leq \infty$. Then $Q^{n} f \in M^{p, q}\left(\mathbb{R}^{2 d}\right)$, for every $n \in \mathbb{N}_{+}$.

Damping of interferences in comparison with the Wigner distribution. This topic is strictly connected with the smoothness of $Q^{n}$, measured using the Fourier Lebesgue wave-front set. It already paved the way for showing the smoothness of the standard Born-Jordan distribution $Q^{1}$ in [12]. Here it is proved that it is the right instrument to measure the smoothness of $Q^{n}$, in comparison with the Wigner distribution, for every $n \in \mathbb{N}_{+}$.

The notion of wave-front set of a distribution is nowadays a standard technique in the study of singularities for solutions to partial differential equations. The basic idea is to detect the location and orientation of the singularities of a distribution $f$ by looking at which directions the Fourier transform of $\varphi f$ fails to decay rapidly, where $\varphi$ is a cut-off function supported in a neighbourhood of any given point $x_{0}$. This test is performed in the framework of edge detection, where often the Fourier transform is replaced by other transforms, see e.g. [37] and the references therein.

We shall use the Fourier-Lebesgue wave-front set, introduced in [40, 41, 42], and related to the Fourier-Lebesgue spaces $\mathcal{F} L_{s}^{q}\left(\mathbb{R}^{d}\right), s \in \mathbb{R}, 1 \leq q \leq \infty$. Recall that the norm in the space $\mathcal{F} L_{s}^{q}\left(\mathbb{R}^{d}\right), 1 \leq q \leq \infty$, is given by

$$
\|f\|_{\mathcal{F} L_{s}^{q}\left(\mathbb{R}^{d}\right)}=\left\|\widehat{f}(\omega)\langle\omega\rangle^{s}\right\|_{L^{q}\left(\mathbb{R}^{d}\right)},
$$

with $\langle\omega\rangle=\left(1+|\omega|^{2}\right)^{1 / 2}$. Inspired by this definition, given a distribution $f \in \mathcal{S}^{\prime}\left(\mathbb{R}^{d}\right)$ its wave-front set $W F_{\mathcal{F} L_{s}^{q}}(f) \subset \mathbb{R}^{d} \times\left(\mathbb{R}^{d} \backslash\{0\}\right)$, is the set of points $\left(x_{0}, \omega_{0}\right) \in \mathbb{R}^{d} \times \mathbb{R}^{d}$, $\omega_{0} \neq 0$, where the following condition is not satisfied: for some cut-off function $\varphi$ (i.e., $\varphi$ is smooth and compactly supported on $\mathbb{R}^{d}$ ), with $\varphi\left(x_{0}\right) \neq 0$, and some open conic neighbourhood $\Gamma \subset \mathbb{R}^{d} \backslash\{0\}$ of $\omega_{0}$ it holds

$$
\left\|\mathcal{F}[\varphi f](\omega)\langle\omega\rangle^{s}\right\|_{L^{q}(\Gamma)}<\infty .
$$

Observe that $W F_{\mathcal{F} L_{s}^{2}}(f)=W F_{H^{s}}(f)$ is the standard $H^{s}$ wave-front set (see [34, Chapter XIII] and Section 2 below). Roughly speaking, $\left(x_{0}, \omega_{0}\right) \notin W F_{\mathcal{F} L_{s}^{q}}(f)$ means that $f$ has regularity $\mathcal{F} L_{s}^{q}$ at $x_{0}$ and in the direction $\omega_{0}$. We are interested in the $\mathcal{F} L_{s}^{q}$ wave-front set of the Born-Jordan distribution of order $n$ of a given signal $f \in L^{2}\left(\mathbb{R}^{d}\right)$.

Here is the mathematical explanation of the $Q^{n}$ 's smoothing effects:

Theorem 1.3. Let $f \in \mathcal{S}^{\prime}\left(\mathbb{R}^{d}\right)$ be a signal, with $W f \in M^{\infty, q}\left(\mathbb{R}^{2 d}\right)$ for some $1 \leq$ $q \leq \infty$. Let $(z, \zeta) \in \mathbb{R}^{2 d} \times \mathbb{R}^{2 d}$, with $\zeta=\left(\zeta_{1}, \zeta_{2}\right)$ satisfying $\zeta_{1} \cdot \zeta_{2} \neq 0$. Then

$$
(z, \zeta) \notin W F_{\mathcal{F} L_{2 n}^{q}}\left(Q^{n} f\right) \text {. }
$$


This means that, if the Wigner distribution $W f$ has local regularity $\mathcal{F} L^{q}$ and some control at infinity, then $Q^{n} f$ is smoother, possessing $s=2 n$ additional derivatives, at least in the directions $\zeta=\left(\zeta_{1}, \zeta_{2}\right)$ satisfying $\zeta_{1} \cdot \zeta_{2} \neq 0$. In dimension $d=1$ this condition reduces to $\zeta_{1} \neq 0$ and $\zeta_{2} \neq 0$. Hence this result explains the smoothing phenomenon of such distributions, which involves all the directions except those of the coordinates axes. That is why the interferences of two components which do not share the same time or frequency localization come out substantially reduced. Observe that for $n=1$ we recapture the damping phenomenon of the classical Born-Jordan distribution (cf. [12, Theorem 1.2]).

For signal in $L^{2}\left(\mathbb{R}^{d}\right)$, the previous result can be rephrased in terms of the Hörmander's wave-front set as follows:

Corollary 1.4. Let $f \in L^{2}\left(\mathbb{R}^{d}\right)$, so that $W f \in L^{2}\left(\mathbb{R}^{2 d}\right)$. Let $(z, \zeta)$ be as in the statement of Theorem 1.3. Then $(z, \zeta) \notin W F_{H^{2 n}}\left(Q^{n} f\right)$, i.e. $Q^{n} f$ has regularity $H^{2 n}$ at $z$ and in the direction $\zeta$.

It seems that the smoothing effects could not occur in the directions $\zeta_{1} \cdot \zeta_{2}=0$, as the evidences in the pictures show. From the mathematical point of view, we provide the explanation below.

Theorem 1.5. Suppose that for some $1 \leq p, q_{1}, q_{2} \leq \infty, n \in \mathbb{N}_{+}$and $C>0$, it occurs

$$
\left\|Q^{n} f\right\|_{M^{p, q_{1}}} \leq C\|W f\|_{M^{p, q_{2}}},
$$

for every $f \in \mathcal{S}\left(\mathbb{R}^{d}\right)$. Then $q_{1} \geq q_{2}$.

In other terms, for a general signal, the BJDn is not everywhere smoother than the Wigner distribution. As expected, the problems arise in the directions $\zeta=$ $\left(\zeta_{1}, \zeta_{2}\right)$ such that $\zeta_{1} \cdot \zeta_{2}=0$.

Visual Comparison in dimension $d=1$ between $Q^{n}$ and the Wigner Distribution. We now illustrate the effect of using higher order cross-term suppression by means of the generalized BJDn. We display the time-frequency distributions of both synthetic and real signals. More precisely, Figure 1 shows a comparison of the Wigner transform, the Born-Jordan transform and generalised Born-Jordan transform of the sum of four rotated Gaussian windows. It is clearly visible, that the amount of cross-term suppression increases by applying higher-order smoothing.

The second example, shown in Figure 2, depicts the Wigner transform, the BornJordan transform and two versions of generalised Born-Jordan transform $(n=10$ and $n=100$ ) of another synthetic signal consisting of two linear chirps. It is notable, that the geometry of this example is different from the previous one in the sense of lacking symmetry around zero.

As a final example, shown in Figure 3, we applied the Wigner transform, the 


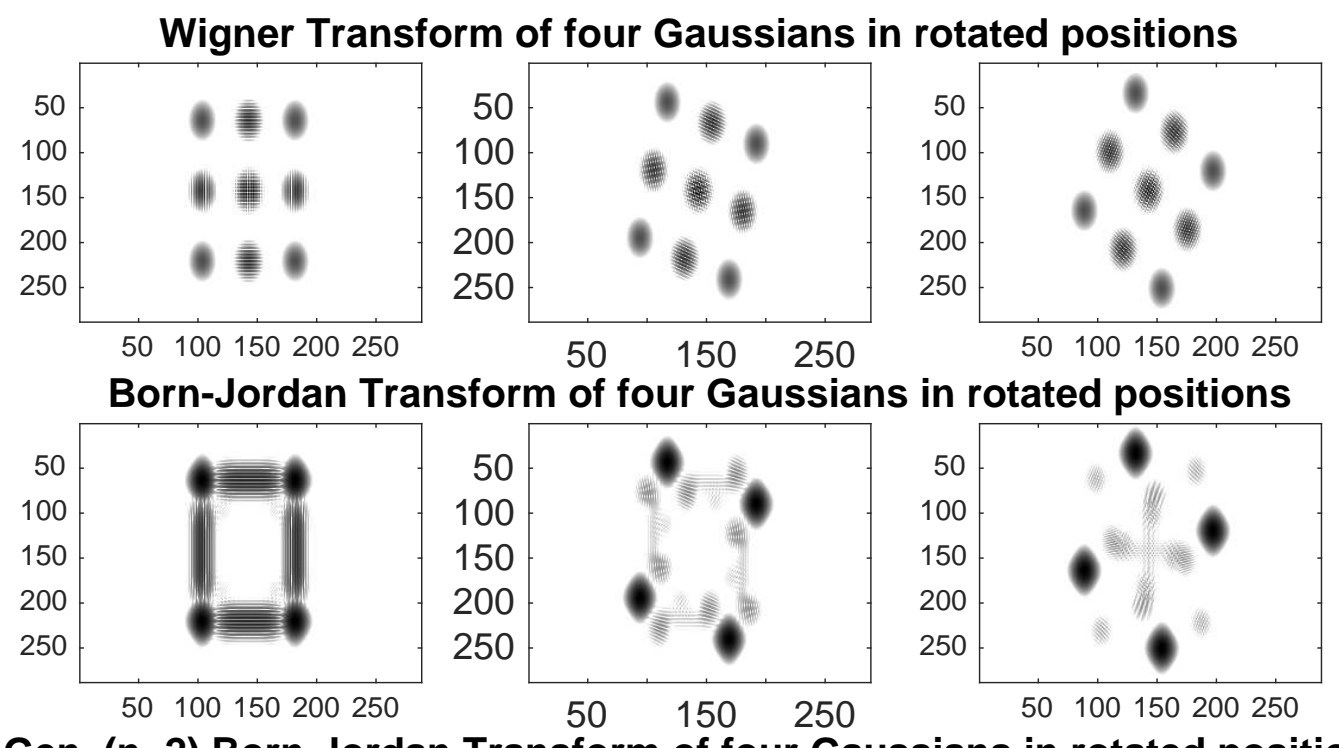

Gen. $(n=2)$ Born-Jordan Transform of four Gaussians in rotated positions
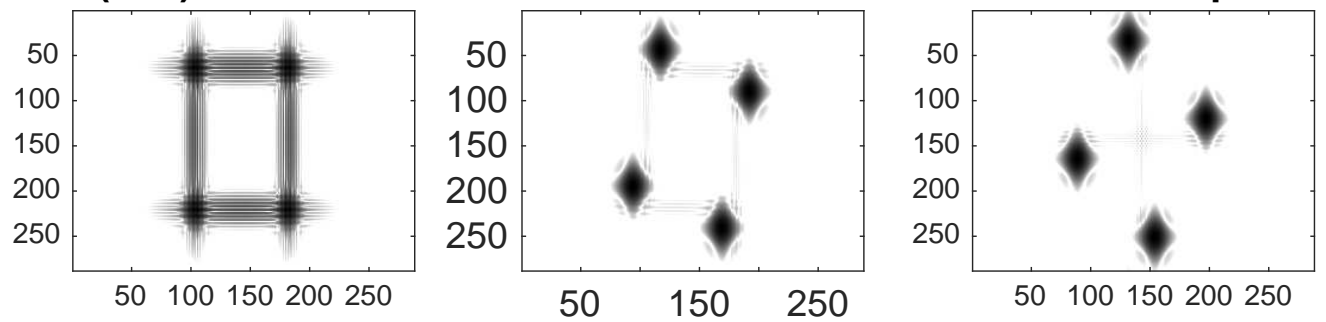

Figure 1. Four Gaussian Windows in rotated positions: Comparison of Wigner distribution, Born-Jordan and generalised BornJordan distribution

Born-Jordan transform and two versions of generalised Born-Jordan transform to a classical real signal, namely a bat call. As in the first example, the suppression of artefacts increases for exponent $n=2$, while, when applying even higher order smoothing, we observe a loss of concentration in time-frequency. As in the case of the two chirps, the geometry of this example lacks symmetry around zero.

The Born-Jordan quantization of order $n$. This procedure arises as the natural extension of the $n=1$ case (that is, the classical Born-Jordan quantization). Observe that choosing $n=0$, we obtain the Weyl quantization. We denote by $\hbar$ the reduced Planck's constant. 

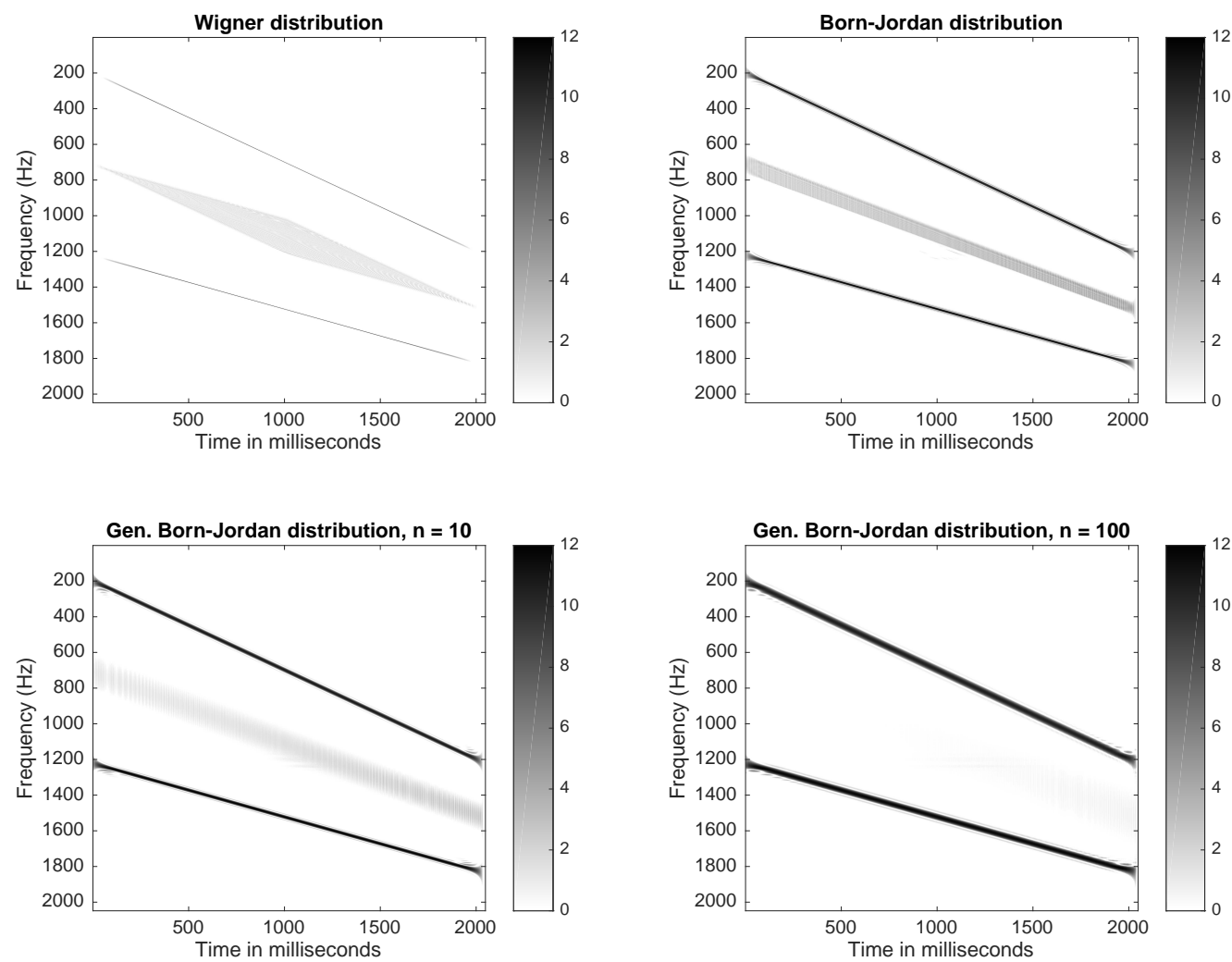

Figure 2. Two linear chirps: Comparison of Wigner distribution, Born-Jordan and generalised Born-Jordan distribution

Definition 1.6. For $n \in \mathbb{N}$, the Born-Jordan quantization of order $n$ is the mapping

$$
a \in \mathcal{S}^{\prime}\left(\mathbb{R}^{2 d}\right) \mapsto \widehat{A}_{\mathrm{BJ}, n}=\mathrm{Op}_{B J, n}(a)=\left(\frac{1}{2 \pi \hbar}\right)^{d} \int_{\mathbb{R}^{2 d}}\left(\mathcal{F}_{\sigma} a\right)(z) \Theta^{n}(z) \widehat{T}(z) d z,
$$

where $\widehat{T}(z)=e^{-2 \pi i \sigma(\widehat{z}, z)}$ is the Heisenberg operator and $\sigma$ the standard symplectic form (see the notation below).

The case $n=0\left(\Theta^{0} \equiv 1\right)$ is the well-known Weyl quantization.

Though, working in the framework of time-frequency analysis, we shall set $\hbar=$ $1 /(2 \pi)$ so that the constant in front of the integrals in (1.14) disappears.

The paper is organized as follows. 

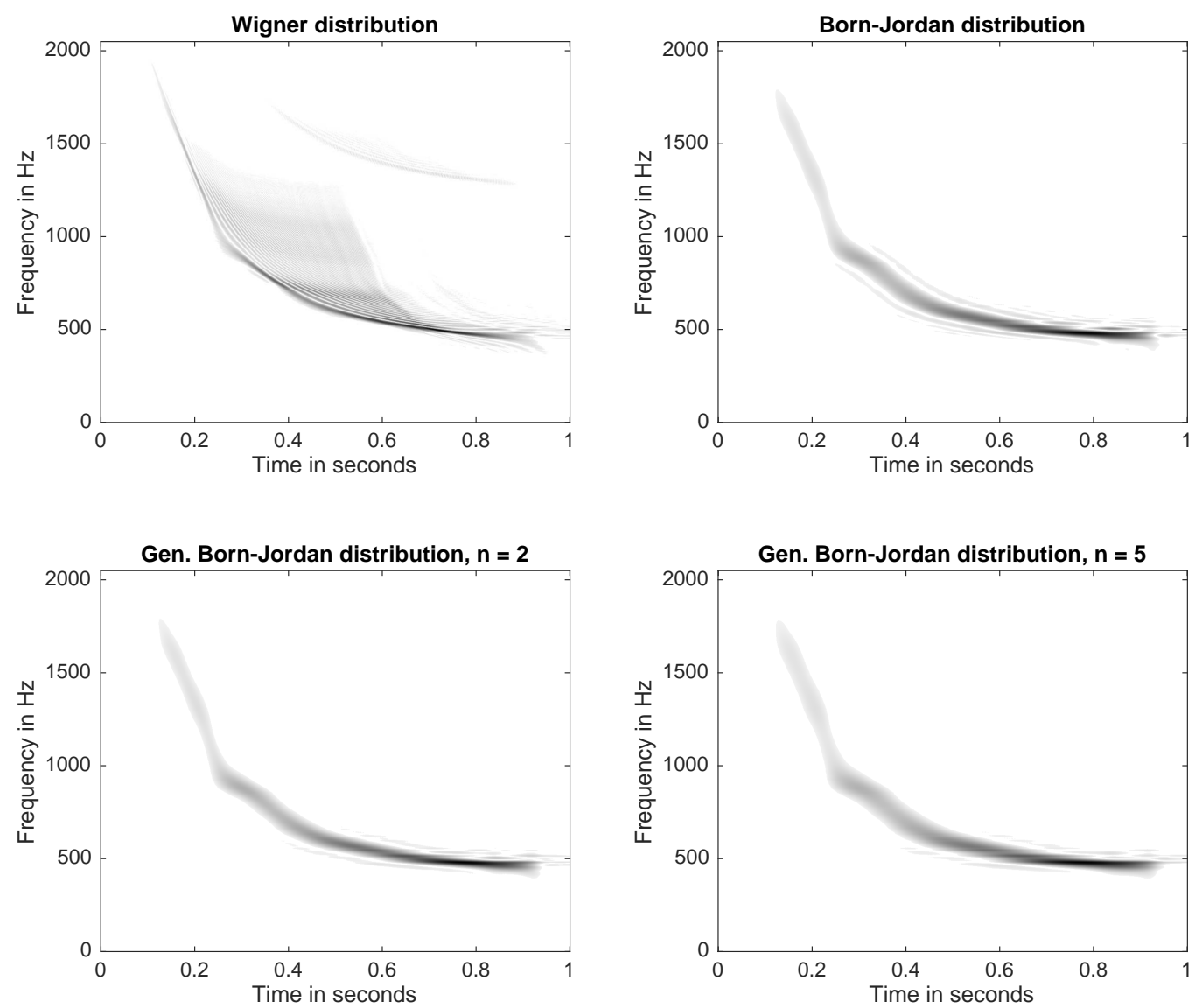

Figure 3. Bat call signal: Comparison of Wigner distribution, Born-Jordan and generalised Born-Jordan distribution

\section{PRELiminaries}

2.1. Notation. We use $x \omega=x \cdot \omega=x_{1} \omega_{1}+\ldots+x_{d} \omega_{d}$ for the scalar product in $\mathbb{R}^{d}$, $\langle\cdot, \cdot\rangle$ for the inner product in $L^{2}\left(\mathbb{R}^{d}\right)$ or for the duality pairing between Schwartz functions and temperate distributions (antilinear in the second argument). Given functions $f, g$, we write $f \lesssim g$ if $f(x) \leq C g(x)$ for every $x$ and some constant $C>0$, and similarly for $\gtrsim$. The notation $f \asymp g$ means $f \lesssim g$ and $f \gtrsim g$.

We write $\mathcal{C}_{c}^{\infty}\left(\mathbb{R}^{d}\right)$ for the class of smooth functions on $\mathbb{R}^{d}$ with compact support.

We denote by $\sigma$ the standard symplectic form on the phase space $\mathbb{R}^{2 d} \equiv \mathbb{R}^{d} \times \mathbb{R}^{d}$; the phase space variable is denoted $z=(x, \omega)$ and the dual variable by $\zeta=\left(\zeta_{1}, \zeta_{2}\right)$. 
By definition $\sigma(z, \zeta)=J z \cdot \zeta=\omega \cdot \zeta_{1}-x \cdot \zeta_{2}$, where

$$
J=\left(\begin{array}{cc}
0_{d \times d} & I_{d \times d} \\
-I_{d \times d} & 0_{d \times d}
\end{array}\right)
$$

The Fourier transform of a function $f(x)$ in $\mathbb{R}^{d}$ is

$$
\mathcal{F} f(\omega)=\widehat{f}(\omega)=\int_{\mathbb{R}^{d}} e^{-2 \pi i x \omega} f(x) d x,
$$

and the symplectic Fourier transform of a function $F(z)$ in the phase space $\mathbb{R}^{2 d}$ is defined by

$$
\mathcal{F}_{\sigma} F(\zeta)=\int_{\mathbb{R}^{2 d}} e^{-2 \pi i \sigma(\zeta, z)} F(z) d z .
$$

The symplectic Fourier transform is an involution, i.e., $\mathcal{F}_{\sigma}\left(\mathcal{F}_{\sigma} F\right)=F$. Moreover, $\mathcal{F}_{\sigma} F(\zeta)=\mathcal{F} F(J \zeta)$.

Observe that $\Theta^{n}\left(J\left(\zeta_{1}, \zeta_{2}\right)\right)=\Theta^{n}\left(\zeta_{1}, \zeta_{2}\right)$ so that

$$
\mathcal{F}_{\sigma}\left(\Theta^{n}\right)=\mathcal{F}\left(\Theta^{n}\right), \quad \forall n \in \mathbb{N}_{+} .
$$

For $s \in \mathbb{R}$ the $L^{2}$-based Sobolev space $H^{s}\left(\mathbb{R}^{d}\right)$ is constituted by the distributions $f \in \mathcal{S}^{\prime}\left(\mathbb{R}^{d}\right)$ such that

$$
\|f\|_{H^{s}}:=\left\|\widehat{f}(\omega)\langle\omega\rangle^{s}\right\|_{L^{2}}<\infty
$$

\subsection{Time-frequency representations and main properties.}

2.2.1. Wigner distribution and ambiguity function [25, 30]. We already defined in Introduction, see (1.1), the Wigner distribution $W f$ of a signal $f \in \mathcal{S}^{\prime}\left(\mathbb{R}^{d}\right)$. In general, we have $W f \in \mathcal{S}^{\prime}\left(\mathbb{R}^{2 d}\right)$. When $f \in L^{2}\left(\mathbb{R}^{d}\right)$ we have $W f \in L^{2}\left(\mathbb{R}^{2 d}\right)$ and in fact it turns out

$$
\|W f\|_{L^{2}\left(\mathbb{R}^{2 d}\right)}=\|f\|_{L^{2}\left(\mathbb{R}^{d}\right)}^{2}
$$

In the sequel we will encounter several times the symplectic Fourier transform of $W f$, which is known as the (radar) ambiguity function Af. We have the formula

$$
A f\left(\zeta_{1}, \zeta_{2}\right)=\mathcal{F}_{\sigma} W f\left(\zeta_{1}, \zeta_{2}\right)=\int_{\mathbb{R}^{d}} f\left(y+\frac{1}{2} \zeta_{1}\right) \overline{f\left(y-\frac{1}{2} \zeta_{1}\right)} e^{-2 \pi i \zeta_{2} y} d y
$$

We refer to [25, Chapter 9] and in particular to [25, Proposition 175] for more details. 
2.2.2. Marginal properties of $Q^{n}$. The members of the Cohen class are also called pseudo-density functions since they are supposed to indicate how the signal density is distributed over time and frequency. The terminology pseudo-density is due to the fact that such distributions in general are not positive functions and can take not only negative but even complex values. In order $Q^{n}$ to be a pseudo-density function, it must satisfy certain requirements. In particular, the marginal densities

$$
\int_{\mathbb{R}^{d}} Q^{n} f(x, \omega) d \omega=|f(t)|^{2}, \quad \int_{\mathbb{R}^{d}} Q^{n} f(x, \omega) d x=|\hat{f}(\omega)|^{2},
$$

for every $f$ in the Schwartz class $\mathcal{S}\left(\mathbb{R}^{d}\right)$. It can be shown (see [35] or [28, Proposition 97]) that those conditions are equivalent to the requirements

$$
\mathcal{F}\left(\Theta^{n}\right)(x, 0)=1, \forall x \in \mathbb{R}^{d}, \quad \mathcal{F}\left(\Theta^{n}\right)(0, \omega)=1, \forall \omega \in \mathbb{R}^{d} .
$$

In this case, using (2.1), (1.8) and (1.7), one sees that are trivially satisfied, since $\operatorname{sinc}^{n}(0)=1$, for every $n \in \mathbb{N}$.

2.2.3. The Moyal identity is not satisfied. In quantum mechanics (but perhaps not really necessary for signal analysis, as already stated by Janssen in [35]) a quite convenient property for Cohen's kernels (1.2) is the validity of Moyal's formula ([14, Theorem 14.2 and 27.15]):

$$
\left\langle Q\left(f_{1}, g_{1}\right), Q\left(f_{2}, g_{2}\right)\right\rangle_{L^{2}\left(\mathbb{R}^{2 d}\right)}=\left\langle f_{1}, f_{2}\right\rangle_{L^{2}\left(\mathbb{R}^{d}\right)}{\overline{\left\langle g_{1}, g_{2}\right\rangle_{L^{2}\left(\mathbb{R}^{d}\right)}}}, \quad f_{1}, f_{2}, g_{1}, g_{2} \in L^{2}\left(\mathbb{R}^{d}\right) .
$$

The Wigner distribution, as well as the STFT and the ambiguity function satisfy (2.7). Though, the BJDn $Q^{n}$, for $n \in \mathbb{N}_{+}$, does not fulfil Moyal's identity. To prove this statement, we use the following characterization (cf. [35, Section 3] and [27]):

Proposition 2.1. A member of the Cohen class, cf. (1.2), satisfies Moyal's formula (2.7) if and only if

$$
|\theta(x, \omega)|=1, \quad \text { for all } \quad(x, \omega) \in \mathbb{R}^{2 d} .
$$

Choosing $Q=Q^{n}, n \in \mathbb{N}_{+}$, we have $\theta(x, \omega)=\operatorname{sinc}^{n}(x \omega)$, so that condition (2.8) is not satisfied for any $n \in \mathbb{N}_{+}$. Observe that for $n=0$ (the Wigner distribution) the previous conditions holds, as expected.

2.3. Modulation spaces [25, 19, 20, 21, 30]. Modulation spaces are used in Timefrequency Analysis to measure the time-frequency concentration of a signal. As already observed in the introduction, their construction relies on the notion of short-time (or windowed) Fourier transform defined in (1.10). 
Let now $s \in \mathbb{R}, 1 \leq p, q \leq \infty$. The modulation space $M_{s}^{p, q}\left(\mathbb{R}^{d}\right)$ consists of all tempered distributions $f \in \mathcal{S}^{\prime}\left(\mathbb{R}^{d}\right)$ such that

$$
\|f\|_{M_{s}^{p, q}}:=\left(\int_{\mathbb{R}^{d}}\left(\int_{\mathbb{R}^{d}}\left|V_{g} f(x, \omega)\right|^{p}\langle\omega\rangle^{s p} d x\right)^{q / p} d \omega\right)^{1 / q}<\infty
$$

(with obvious changes for $p=\infty$ or $q=\infty$ ). When $s=0$ we write $M^{p, q}\left(\mathbb{R}^{d}\right)$ instead of $M_{0}^{p, q}\left(\mathbb{R}^{d}\right)$. The shorthand notation for $M_{s}^{p, p}\left(\mathbb{R}^{d}\right)$ is $M_{s}^{p}\left(\mathbb{R}^{d}\right)$. The spaces $M_{s}^{p, q}\left(\mathbb{R}^{d}\right)$ are Banach spaces for any $1 \leq p, q \leq \infty$, and every non-zero $g \in \mathcal{S}\left(\mathbb{R}^{d}\right)$ yields an equivalent norm in (2.9).

Modulation spaces generalize and include as special cases several function spaces arising in Harmonic Analysis. In particular for $p=q=2$ we have

$$
M_{s}^{2}\left(\mathbb{R}^{d}\right)=H^{s}\left(\mathbb{R}^{d}\right),
$$

whereas $M^{1}\left(\mathbb{R}^{d}\right)$ coincides with the Segal algebra $S_{0}\left(\mathbb{R}^{d}\right)$ (cf. [17]), and $M^{\infty, 1}\left(\mathbb{R}^{d}\right)$ is the so-called Sjöstrand class 31.

As already observed in the Introduction, in the notation $M_{s}^{p, q}$ the exponent $p$ is a measure of decay at infinity (on average) in the scale of spaces $\ell^{p}$, whereas the exponent $q$ is a measure of smoothness in the scale $\mathcal{F} L^{q}$. The number $s$ is a further regularity index, completely analogous to that appearing in the Sobolev spaces $H^{s}\left(\mathbb{R}^{d}\right)$.

Other modulation spaces, also known as Wiener amalgam spaces, are obtained by exchanging the order of integration in (2.9). Precisely, the modulation spaces $W\left(\mathcal{F} L^{p}, L^{q}\right)\left(\mathbb{R}^{d}\right)$, for $p, q \in[1,+\infty)$, is given by the distributions $f \in \mathcal{S}^{\prime}\left(\mathbb{R}^{d}\right)$ such that

$$
\|f\|_{W\left(\mathcal{F} L^{p}, L^{q}\right)\left(\mathbb{R}^{d}\right)}:=\left(\int_{\mathbb{R}^{d}}\left(\int_{\mathbb{R}^{d}}\left|V_{g} f(x, \omega)\right|^{p} d \omega\right)^{q / p} d x\right)^{1 / q}<\infty
$$

(with obvious changes for $p=\infty$ or $q=\infty$ ). Using Parseval identity in (1.10), we can write the so-called fundamental identity of time-frequency analysis

$$
V_{g} f(x, \omega)=e^{-2 \pi i x \omega} V_{\hat{g}} \hat{f}(\omega,-x),
$$

hence

$$
\left|V_{g} f(x, \omega)\right|=\left|V_{\hat{g}} \hat{f}(\omega,-x)\right|=\left|\mathcal{F}\left(\hat{f} T_{\omega} \overline{\hat{g}}\right)(-x)\right|
$$

so that

$$
\|f\|_{M^{p, q}}=\left(\int_{\mathbb{R}^{d}}\left\|\hat{f} T_{\omega} \overline{\hat{g}}\right\|_{\mathcal{F} L^{p}}^{q} d \omega\right)^{1 / q}=\|\hat{f}\|_{W\left(\mathcal{F} L^{p}, L^{q}\right)} .
$$

This means that such Wiener amalgam spaces can be viewed as the image under Fourier transform of modulation spaces: $\mathcal{F}\left(M^{p, q}\right)=W\left(\mathcal{F} L^{p}, L^{q}\right)$. 
We will frequently use the following product property of Wiener amalgam spaces ([19, Theorem $1(\mathrm{v})])$ : For $1 \leq p, q \leq \infty$,

$$
\text { if } f \in W\left(\mathcal{F} L^{1}, L^{\infty}\right) \text { and } g \in W\left(\mathcal{F} L^{p}, L^{q}\right) \text { then } f g \in W\left(\mathcal{F} L^{p}, L^{q}\right) \text {. }
$$

Taking $p=1, q=\infty$, we obtain that $W\left(\mathcal{F} L^{1}, L^{\infty}\right)\left(\mathbb{R}^{2 d}\right)$ is an algebra under pointwise multiplication.

Proposition 2.2. Let $1 \leq p, q \leq \infty$ and $A \in G L(d, \mathbb{R})$. Then, for every $f \in$ $W\left(\mathcal{F} L^{p}, L^{q}\right)\left(\mathbb{R}^{d}\right)$,

$$
\|f(A \cdot)\|_{W\left(\mathcal{F} L^{p}, L^{q}\right)} \leq C|\operatorname{det} A|^{(1 / p-1 / q-1)}\left(\operatorname{det}\left(I+A^{*} A\right)\right)^{1 / 2}\|f\|_{W\left(\mathcal{F} L^{p}, L^{q}\right)} .
$$

In particular, for $A=\lambda I, \lambda>0$,

$$
\|f(A \cdot)\|_{W\left(\mathcal{F} L^{p}, L^{q}\right)} \leq C \lambda^{d\left(\frac{1}{p}-\frac{1}{q}-1\right)}\left(\lambda^{2}+1\right)^{d / 2}\|f\|_{W\left(\mathcal{F} L^{p}, L^{q}\right)} .
$$

In the proof of Theorem 1.5 we will use dilation properties of Gaussians (first proved in [46, Lemma 1.8], see also [9, Lemma 3.2]):

Lemma 2.3. Let $\varphi(x)=e^{-\pi|x|^{2}}$ and $\lambda>0$. Then

$$
\|\varphi(\lambda \cdot)\|_{M^{p, q}} \asymp \lambda^{-d / q^{\prime}} \quad \text { as } \lambda \rightarrow+\infty .
$$

2.4. Wave-front set for Fourier-Lebesgue spaces [34, 40]. The notion of $H^{s}$ wave-front set allows to quantify the regularity of a function or distribution in the Sobolev scale, at any given point and direction. This is done by microlocalizing the definition of the $H^{s}$ norm in (2.2) as follows (cf. [34, Chapter XIII]).

Given a distribution $f \in \mathcal{S}^{\prime}\left(\mathbb{R}^{d}\right)$ we define its wave-front set $W F_{H^{s}}(f) \subset \mathbb{R}^{d} \times$ $\left(\mathbb{R}^{d} \backslash\{0\}\right)$, as the set of points $\left(x_{0}, \omega_{0}\right) \in \mathbb{R}^{d} \times \mathbb{R}^{d}, \omega_{0} \neq 0$, where the following condition is not satisfied: for some cut-off function $\varphi \in C_{c}^{\infty}\left(\mathbb{R}^{d}\right)$ with $\varphi\left(x_{0}\right) \neq 0$ and some open conic neighborhood of $\Gamma \subset \mathbb{R}^{d} \backslash\{0\}$ of $\omega_{0}$ we have

$$
\left\|\mathcal{F}[\varphi f](\omega)\langle\omega\rangle^{s}\right\|_{L^{2}(\Gamma)}<\infty .
$$

More in general one can start from the Fourier-Lebesgue spaces $\mathcal{F} L_{s}^{q}\left(\mathbb{R}^{d}\right), s \in \mathbb{R}$, $1 \leq q \leq \infty$, which is the space of distributions $f \in \mathcal{S}^{\prime}\left(\mathbb{R}^{d}\right)$ such that the norm in (1.11) is finite. Arguing exactly as above (with the space $L^{2}$ replaced by $L^{q}$ ) one then arrives in a natural way to a corresponding notion of wave-front set $W F_{\mathcal{F} L_{s}^{q}}(f)$ as we anticipated in Introduction (see (1.12)).

For our purpose we need to recall some basic results about the action of constant coefficient linear partial differential operators on such wave-front set (cf. [40]).

Given the operator

$$
P=\sum_{|\alpha| \leq m} c_{\alpha} \partial^{\alpha}, \quad c_{\alpha} \in \mathbb{C}
$$

it is straightforward to see that, for $1 \leq q \leq \infty, s \in \mathbb{R}, f \in \mathcal{S}^{\prime}\left(\mathbb{R}^{d}\right)$,

$$
W F_{\mathcal{F} L_{s}^{q}}(P f) \subset W F_{\mathcal{F} L_{s+m}^{q}}(f) .
$$


Consider now the inverse inclusion. We say that $\zeta \in \mathbb{R}^{d}, \zeta \neq 0$, is non characteristic for the operator $P$ if

$$
\sum_{|\alpha|=m} c_{\alpha} \zeta^{\alpha} \neq 0
$$

This means that $P$ is elliptic in the direction $\zeta$. The following result is a microlocal version of the classical regularity result of elliptic operators (see [40, Corollary 1 $(2)])$ :

Proposition 2.4. Let $1 \leq q \leq \infty, s \in \mathbb{R}$ and $f \in \mathcal{S}^{\prime}\left(\mathbb{R}^{d}\right)$. Let $z \in \mathbb{R}^{d}$ and suppose that $\zeta \in \mathbb{R}^{d} \backslash\{0\}$ is non characteristic for $P$. Then, if $(z, \zeta) \notin W F_{\mathcal{F} L_{s}^{q}}(P f)$ we have $(z, \zeta) \notin W F_{\mathcal{F} L_{s+m}^{q}}(f)$.

\section{Generalized Born-Jordan Kernels for Monomials}

Let $\mathbb{C}[x, \omega]$ be the commutative ring of polynomials generated by $x$ and $\omega$; it consists of all finite sums $a(x, \omega)=\sum \lambda_{m \ell} a_{m \ell}(x, \omega)\left(\lambda_{m \ell} \in \mathbb{C}\right)$ where $a_{m \ell}(x, \omega)=$ $\omega^{m} x^{\ell}$ with $(m, \ell) \in \mathbb{N}^{2}$. We identify $\mathbb{C}[x, \omega]$ with the ring of polynomial functions in the variables $(x, \omega) \in \mathbb{R}^{2}$. We denote by $\mathbb{C}[\widehat{x}, \widehat{\omega}]$ the corresponding Weyl algebra; it is realized as the non-commutative unital algebra generated by the two operators $\widehat{x}$ and $\widehat{\omega}$ satisfying $[\widehat{x}, \widehat{\omega}]=(i / 2 \pi) I_{\mathrm{d}}$. These operators are realized as the unbounded operators defined on $L^{2}(\mathbb{R})$ by $\widehat{x} f=x f$ and $\widehat{\omega} f=-(i / 2 \pi) \partial_{x} f$. We will call quantization of $\mathbb{C}[x, \omega]$ any continuous linear mapping Op : $\mathbb{C}[x, \omega] \longrightarrow \mathbb{C}[\widehat{x}, \widehat{\omega}]$ having the following properties:

(Q1) Triviality: $\mathrm{Op}(1)=I_{\mathrm{d}}, \mathrm{Op}(x)=\widehat{x}$, and $\mathrm{Op}(\omega)=\widehat{\omega}$;

(Q2) Dirac's restricted rule:

$$
\left[x, \mathrm{Op}\left(a_{m \ell}\right)\right]=(i / 2 \pi) \mathrm{Op}\left(\left\{x, a_{m \ell}\right\}\right), \quad\left[\omega, \mathrm{Op}\left(a_{m \ell}\right)\right]=(i / 2 \pi) \mathrm{Op}\left(\left\{\omega, a_{m \ell}\right\}\right) ;
$$

(Q3) Self-adjointness: If $a \in \mathbb{C}[x, \omega]$ then $\operatorname{Op}(a)$ is self-adjoint on its domain.

One shows [15] (also see [8]) that for every quantization of $\mathbb{C}[x, \omega]$ there exists [8, 15] a function $f$ with $f(0)=1$ and $e^{-i t / 2} f$ real such that

$$
\mathrm{Op}\left(a_{m \ell}\right)=\sum_{j=0}^{\min (m, \ell)} j !\left(\begin{array}{l}
m \\
j
\end{array}\right)\left(\begin{array}{l}
\ell \\
j
\end{array}\right) f^{(j)}(0)(2 \pi)^{-j} \widehat{\omega}^{m-j} \widehat{x}^{\ell-j} .
$$

Let $\left(a_{m \ell}\right)_{\sigma}=\mathcal{F}_{\sigma} a_{m \ell}$ be the symplectic Fourier transform of $a_{m \ell}$ and $\widehat{T}(z)=$ $e^{-2 \pi i \sigma(\widehat{z}, z)}$ the Heisenberg operator.

Proposition 3.1. Let Op : $\mathcal{S}^{\prime}\left(\mathbb{R}^{2 n}\right) \longrightarrow \mathcal{L}\left(\mathcal{S}\left(\mathbb{R}^{n}\right), \mathcal{S}^{\prime}\left(\mathbb{R}^{n}\right)\right)$ be a quantization having the properties (Q1), (Q2), (Q3). (i) The restriction of Op to $\mathbb{C}[x, \omega]$ is then given by

$$
\operatorname{Op}\left(a_{m \ell}\right)=\int\left(a_{m \ell}\right)_{\sigma}(x, \omega) \Phi(2 \pi \omega x) \widehat{T}(x, \omega) d \omega d x
$$


where $\Phi(t)=e^{-i t / 2} f(t)$. (ii) The Cohen kernel $\theta$ of Op thus has symplectic Fourier transform $\mathcal{F}_{\sigma} \theta$ given by

$$
\mathcal{F}_{\sigma} \theta(x, \omega)=\Phi(2 \pi \omega x) .
$$

Proof. A detailed proof is given in by Domingo and Galapon [15] (formulas (10) and (14)). Notice that formula (3.2) readily follows from (3.1) using the elementary formula

$$
\mathcal{F}\left(\omega^{m} \otimes x^{\ell}\right)=(i / 2 \pi)^{m+\ell} \delta^{(m)}(\omega) \otimes \delta^{(\ell)}(x) .
$$

Formula (3.3) follows since (3.2) is the Weyl representation of the operator with twisted symbol $\left(a_{m \ell}\right)_{\sigma} \Phi$ [the twisted symbol is the symplectic Fourier transform of the usual symbol].

Remark 3.2. This result shows that if one limits oneself to pseudo-differential calculi satisfying the Dirac conditions (Q2) then the Cohen kernel is of a very particular type: its Fourier transform only depends on the product $\omega x$. In particular, the associated quasidistribution $Q \psi=W \psi * \theta$ satisfies the marginal conditions since $\mathcal{F}_{\sigma} \theta(0)=\Phi(0)=1$ (see [27], formula (7.29), p. 107).

We now focus on the case the symplectic Fourier transform of the Cohen kernel is given by

$$
\mathcal{F}_{\sigma} \theta(x, \omega)=\operatorname{sinc}^{n}(\pi \omega x), n \in \mathbb{N}=\{0,1,2, \ldots\} .
$$

With the notation above we thus have $\Phi(\pi \omega x)=\operatorname{sinc}^{n}(\pi \omega x)$ so that $\Phi(t)=$ $\operatorname{sinc}^{n}(t / 2)$ and hence $f(t)=e^{i t / 2} \operatorname{sinc}^{n}(t / 2)$. Suppose first $n=0$; then $f^{(j)}(0)=$ $(i / 2)^{j}$ hence formula (3.1) yields

$$
\mathrm{Op}\left(a_{m \ell}\right)=\sum_{j=0}^{\min (m, \ell)}\left(\begin{array}{l}
m \\
j
\end{array}\right)\left(\begin{array}{l}
\ell \\
j
\end{array}\right) j !\left(\frac{i}{4 \pi}\right)^{j} \widehat{\omega}^{m-j} \widehat{x}^{\ell-j}
$$

so that $\mathrm{Op}\left(a_{m \ell}\right)=\mathrm{Op}^{\mathrm{W}}\left(a_{m \ell}\right)=\mathrm{Op}_{B J, 0}\left(a_{m \ell}\right)$ (see (1.14) $)$ is just the Weyl ordering of the monomial $a_{m \ell}$ ([15] and [27], p.34). Suppose next $n=1$. Then $f^{(j)}(0)=$ $i^{j} /(j+1)$ and

$$
\mathrm{Op}\left(a_{m \ell}\right)=\sum_{j=0}^{\min (m, \ell)}\left(\begin{array}{c}
m \\
j
\end{array}\right)\left(\begin{array}{l}
\ell \\
j
\end{array}\right) \frac{j !}{j+1}\left(\frac{i}{2 \pi}\right)^{j} \widehat{\omega}^{m-j} \widehat{x}^{\ell-j}
$$

here $\mathrm{Op}\left(a_{m \ell}\right)=\mathrm{Op}_{B J, 1}\left(a_{m \ell}\right)$ is the Born-Jordan ordering ([15] and [27, page 34]).

In the case of a general $n$ we have, by Leibniz's formula,

$$
f^{(j)}(0)=\sum_{k=0}^{j}\left(\begin{array}{l}
j \\
k
\end{array}\right)\left(\frac{i}{2}\right)^{j-k}\left(\frac{1}{2}\right)^{k}\left(\frac{d^{k}}{d t^{k}} \operatorname{sinc}^{n}\right)(0) .
$$


The derivatives of $\operatorname{sinc}^{n}$ at $t=0$ can be calculated using Faà di Bruno's formula [16] for the derivatives of the composition of two functions

$$
(g \circ h)^{(k)}(t)=\sum_{\kappa \cdot \alpha=k}\left(\begin{array}{l}
k \\
\alpha
\end{array}\right) g^{(|\alpha|)}(h(t)) \Pi_{\alpha}(t)
$$

where $\kappa=(1,2, \ldots, k), \alpha=\left(\alpha_{1}, \alpha_{2}, \ldots, \alpha_{k}\right) \in \mathbb{N}^{k}$ and

$$
\Pi_{\alpha}(t)=\left(\frac{1}{1 !} h^{(1)}(t)\right)^{\alpha_{1}}\left(\frac{1}{2 !} h^{(2)}(t)\right)^{\alpha_{2}} \cdots\left(\frac{1}{k !} h^{(k)}(t)\right)^{\alpha_{k}} .
$$

Choosing $g(t)=x^{n}$ and $h(t)=\operatorname{sinc}(t / 2)$ this formula yields

$$
\frac{d^{k}}{d t^{k}} \operatorname{sinc}^{n}(0)=\sum_{\substack{\kappa \cdot \alpha=k \\
|\alpha| \leq n}}\left(\begin{array}{c}
k \\
\alpha
\end{array}\right)\left(\begin{array}{c}
n \\
|\alpha|
\end{array}\right)|\alpha| ! \Pi_{\alpha}(0) ;
$$

since $\operatorname{sinc}^{(2 m+1)}(0)=0$ and $\operatorname{sinc}^{(2 m)}(0)=(-1)^{m} /(2 m+1)$ we have

$$
\Pi_{\alpha}(0)=\frac{1}{1 !\left(\alpha_{1}+1\right)^{\alpha_{1}} 2 !\left(\alpha_{2}+1\right)^{\alpha_{2}} \cdots k !\left(\alpha_{k}+1\right)^{\alpha_{k}}} .
$$

\section{Time-Frequency Analysis of the NTH Born-Jordan Kernel}

The Born-Jordan kernel $\Theta(\zeta)$ in (1.3) belongs to the space $W\left(\mathcal{F} L^{1}, L^{\infty}\right)\left(\mathbb{R}^{2 d}\right)$, as proved in [12]:

Proposition 4.1. The function $\Theta^{1}$ in $(1.3)$ belongs to $W\left(\mathcal{F} L^{1}, L^{\infty}\right)\left(\mathbb{R}^{2 d}\right)$.

The previous property is true for any $\Theta^{n}, n \in \mathbb{N}_{+}$, as shown below.

Proposition 4.2. For $n \in \mathbb{N}_{+}$, the function $\Theta^{n}$ defined in (1.7) belongs to the Wiener algebra $W\left(\mathcal{F} L^{1}, L^{\infty}\right)\left(\mathbb{R}^{2 d}\right)$.

Proof. The result is attained by induction on $n$. We know that $\Theta^{1} \in W\left(\mathcal{F} L^{1}, L^{\infty}\right)\left(\mathbb{R}^{2 d}\right)$ by Proposition 4.1. If we assume $\Theta^{n} \in W\left(\mathcal{F} L^{1}, L^{\infty}\right)\left(\mathbb{R}^{2 d}\right)$, for a certain integer $n>1$, we obtain

$$
\Theta^{n+1}=\Theta^{n} \cdot \Theta^{1} \in W\left(\mathcal{F} L^{1}, L^{\infty}\right)\left(\mathbb{R}^{2 d}\right) \cdot W\left(\mathcal{F} L^{1}, L^{\infty}\right)\left(\mathbb{R}^{2 d}\right) \hookrightarrow W\left(\mathcal{F} L^{1}, L^{\infty}\right)\left(\mathbb{R}^{2 d}\right),
$$

since the Banach space $W\left(\mathcal{F} L^{1}, L^{\infty}\right)\left(\mathbb{R}^{2 d}\right)$ is an algebra by pointwise product. This gives the claim.

In [12] it was shown the following property for the chirp function:

Proposition 4.3. The function $F\left(\zeta_{1}, \zeta_{2}\right)=e^{2 \pi i \zeta_{1} \zeta_{2}}$ belongs to $W\left(\mathcal{F} L^{1}, L^{\infty}\right)\left(\mathbb{R}^{2 d}\right)$. 
Since $W\left(\mathcal{F} L^{1}, L^{\infty}\right)\left(\mathbb{R}^{2 d}\right)$ can be characterized as the space of pointwise multipliers on the Feichtinger algebra $W\left(\mathcal{F} L^{1}, L^{1}\right)\left(\mathbb{R}^{2 d}\right)[22$, Corollary 3.2.10], the result in Proposition 4.3 could also be deduced from general results about the action of second order characters on the Feichtinger algebra, cf. [17, 44].

By Proposition 4.3 and by the dilation properties for Wiener amalgam spaces (2.11) we can state:

Corollary 4.4. For $\zeta=\left(\zeta_{1}, \zeta_{2}\right)$, consider the function $F_{J}(\zeta)=F(J \zeta)=e^{-2 \pi i \zeta_{1} \zeta_{2}}$. Then $F_{J} \in W\left(\mathcal{F} L^{1}, L^{\infty}\right)\left(\mathbb{R}^{2 d}\right)$.

\section{Smoothness of the Born-Jordan distribution of order $n$}

In the present section we compare the smoothness of the Born-Jordan distribution of order $n$ with the Wigner distribution. In particular we prove Theorem 1.3 . 1.2 .

We begin with the following global result, which in particular implies Theorem

Theorem 5.1. Let $f \in \mathcal{S}^{\prime}\left(\mathbb{R}^{d}\right)$ be a signal, with $W f \in M^{p, q}\left(\mathbb{R}^{2 d}\right)$ for some $1 \leq$ $p, q \leq \infty$. Then

$$
Q^{n} f \in M^{p, q}\left(\mathbb{R}^{2 d}\right)
$$

and moreover

$$
\left(\nabla_{x} \cdot \nabla_{\omega}\right)^{n} Q^{n} f \in M^{p, q}\left(\mathbb{R}^{2 d}\right) .
$$

Here we used the notation

$$
\nabla_{x} \cdot \nabla_{\omega}:=\sum_{j=1}^{d} \frac{\partial^{2}}{\partial x_{j} \partial \omega_{j}} .
$$

Proof. We first show $Q^{n} f \in M^{p, q}\left(\mathbb{R}^{2 d}\right)$. Taking the symplectic Fourier transform in (1.4) we are reduced to prove that

$$
\Theta^{n} \mathcal{F}_{\sigma}(W f)=\Theta^{n} A f \in W\left(\mathcal{F} L^{p}, L^{q}\right)
$$

where $\mathcal{F}_{\sigma}(W f)=A f$ is the ambiguity function of $f$ in (2.4). The claim is attained using the product property (2.10): by Proposition 4.2, the function $\Theta^{n}$ is in $W\left(\mathcal{F} L^{1}, L^{\infty}\right)$ and by assumption $W f \in M^{p, q}\left(\mathbb{R}^{2 d}\right)$ so that $\mathcal{F}(W f) \in W\left(\mathcal{F} L^{p}, L^{q}\right)$, and therefore $\mathcal{F}_{\sigma}(W f)(\zeta)=\mathcal{F}(W f)(J \zeta) \in W\left(\mathcal{F} L^{p}, L^{q}\right)$ by Proposition 2.2.

We now prove (5.1). Taking the symplectic Fourier transform we see that it is sufficient to prove that

$$
\left(\zeta_{1} \zeta_{2}\right)^{n} \operatorname{sinc}^{n}\left(\zeta_{1} \zeta_{2}\right) \mathcal{F}_{\sigma} W f=\frac{1}{\pi^{n}} \sin ^{n}\left(\pi \zeta_{1} \zeta_{2}\right) \mathcal{F}_{\sigma} W f \in W\left(\mathcal{F} L^{p}, L^{q}\right)
$$


We have,

$$
\sin \left(\pi \zeta_{1} \zeta_{2}\right)=\frac{e^{\pi i \zeta_{1} \zeta_{2}}-e^{-\pi i \zeta_{1} \zeta_{2}}}{2 i} \in W\left(\mathcal{F} L^{1}, L^{\infty}\right)
$$

by Proposition 4.3, Corollary 4.4 and Proposition 2.2 , with the scaling $\lambda=1 / \sqrt{2}$.

Hence, for $n=1$,

$$
\frac{1}{\pi} \sin \left(\pi \zeta_{1} \zeta_{2}\right) \mathcal{F}_{\sigma} W f \in W\left(\mathcal{F} L^{p}, L^{q}\right)
$$

by the product property (2.10). Assume now that, for a certain $n \in \mathbb{N}_{+}$,

$$
\frac{1}{\pi^{n}} \sin ^{n}\left(\pi \zeta_{1} \zeta_{2}\right) \mathcal{F}_{\sigma} W f \in W\left(\mathcal{F} L^{p}, L^{q}\right)
$$

Then

$$
\frac{1}{\pi^{n+1}} \sin ^{n+1}\left(\pi \zeta_{1} \zeta_{2}\right) \mathcal{F}_{\sigma} W f=\underbrace{\frac{1}{\pi} \sin \left(\pi \zeta_{1} \zeta_{2}\right)}_{\in W\left(\mathcal{F} L^{1}, L^{\infty}\right)} \cdot \underbrace{\frac{1}{\pi^{n}} \sin ^{n}\left(\pi \zeta_{1} \zeta_{2}\right) \mathcal{F} \sigma W f}_{\in W\left(\mathcal{F} L^{p}, L^{q}\right)} \in W\left(\mathcal{F} L^{p}, L^{q}\right),
$$

by (5.2) and the product property (2.10) again. By induction we attain the result.

We are now ready to prove Theorem 1.3 .

Proof of Theorem 1.3. Consider $n \in \mathbb{N}_{+}$. We will apply Proposition 2.4 to the $2 n$ th order operator $P^{n}$, where $P=\nabla_{x} \cdot \nabla_{\omega}$ in $\mathbb{R}^{2 d}$. The non characteristic directions for $P^{n}$ are given by the vectors $\zeta=\left(\zeta_{1}, \zeta_{2}\right) \in \mathbb{R}^{d} \times \mathbb{R}^{d}$, satisfying $\zeta_{1} \cdot \zeta_{2} \neq 0$. By (5.1) (with $p=\infty$ ) we have

$$
W F_{\mathcal{F} L^{q}}\left(P^{n} Q^{n} f\right)=\emptyset,
$$

because $\varphi F \in \mathcal{F} L^{q}$ if $\varphi \in C_{c}^{\infty}\left(\mathbb{R}^{2 d}\right)$ and $F \in M^{\infty, q}\left(\mathbb{R}^{2 d}\right)$ (here $\left.F=P^{n} Q^{n} f\right)$. Hence we obtain

$$
(z, \zeta) \notin W F_{\mathcal{F} L^{q}}\left(P^{n} Q^{n} f\right), \quad \forall(z, \zeta) \text { such that } \zeta=\left(\zeta_{1}, \zeta_{2}\right), \zeta_{1} \cdot \zeta_{2} \neq 0 .
$$

Since $\zeta$ is non characteristic for the operator $P^{n}$, by Proposition 2.4 we infer

$$
(z, \zeta) \notin W F_{\mathcal{F} L_{2 n}^{q}}\left(Q^{n} f\right)
$$

for every $z \in \mathbb{R}^{2 d}$.

Proof of Corollary 1.4. Apply Theorem 1.3 with $q=2$. Indeed, for $f \in L^{2}\left(\mathbb{R}^{d}\right)$ Moyal's formula gives $W f \in L^{2}\left(\mathbb{R}^{2 d}\right)=M^{2,2}\left(\mathbb{R}^{d}\right) \subset M^{\infty, 2}\left(\mathbb{R}^{2 d}\right)$ (cf. (2.3) $)$. Observe that the $\mathcal{F} L_{2 n}^{2}$ wave-front set coincides with the $H^{2 n}$ wave-front set.

The proof of Theorem 1.5 requires Lemma 5.1 in [12]:

Lemma 5.2. Let $\chi \in C_{c}^{\infty}(\mathbb{R})$. Then the function $\chi\left(\zeta_{1} \zeta_{2}\right)$ belongs to $W\left(\mathcal{F} L^{1}, L^{\infty}\right)\left(\mathbb{R}^{2 d}\right)$. 
As announced in the introduction, the smoothing phenomena of the $Q^{n}$ distributions do not involve the whole phase space. We do not have any gain in the directions $\zeta_{1} \cdot \zeta_{2}=0$ as it comes up clearly from the proof of the following issue.

Proof of Theorem [1.5. The pattern is similar to that of Theorem 1.4 in [12]. We detail the main steps for sake of clarity. The idea is to test the estimate (1.13) using rescaled Gaussian functions $f(x)=\varphi(\lambda x)$, with $\lambda>0$ large parameter. We shall prove that, restricting to a neighbourhood of $\zeta_{1} \cdot \zeta_{2}=0$, the constrain $q_{1} \geq q_{2}$ must be satisfied.

An easy computation (see e.g. [30, Formula (4.20)]) yields

$$
W(\varphi(\lambda \cdot))(x, \omega)=2^{d / 2} \lambda^{-d} \varphi(\sqrt{2} \lambda x) \varphi\left(\sqrt{2} \lambda^{-1} \omega\right) .
$$

For every $1 \leq p, q \leq \infty$, the above formula gives

$$
\|W(\varphi(\lambda \cdot))\|_{M^{p, q}}=2^{d / 2} \lambda^{-d}\|\varphi(\sqrt{2} \lambda \cdot)\|_{M^{p, q}}\left\|\varphi\left(\sqrt{2} \lambda^{-1} \cdot\right)\right\|_{M^{p, q}} .
$$

By the dilation properties of Gaussians in Lemma 2.3

$$
\|W(\varphi(\lambda \cdot))\|_{M^{p, q}} \asymp \lambda^{-2 d+d / q+d / p} \text { as } \lambda \rightarrow+\infty .
$$

We now study the $M^{p, q}$-norm of the BJDn $Q^{n}(\varphi(\lambda \cdot))$. The idea is to estimate such a norm from below obtaining the same expansion as in (5.4).

$$
\left\|Q^{n}(\varphi(\lambda \cdot))\right\|_{M^{p, q}}=\left\|\mathcal{F}_{\sigma}\left(\Theta^{n}\right) * W(\varphi(\lambda \cdot))\right\|_{M^{p, q}} .
$$

By taking the symplectic Fourier transform and using Lemma 5.2 and the product property (2.10) we have

$$
\begin{aligned}
\left\|\mathcal{F}_{\sigma}\left(\Theta^{n}\right) * W(\varphi(\lambda \cdot))\right\|_{M^{p, q}} & \asymp\left\|\Theta^{n} \mathcal{F}_{\sigma}[W(\varphi(\lambda \cdot))]\right\|_{W\left(\mathcal{F} L^{p}, L^{q}\right)} \\
& \gtrsim\left\|\Theta^{n}\left(\zeta_{1}, \zeta_{2}\right) \chi\left(\zeta_{1} \zeta_{2}\right) \mathcal{F}_{\sigma}[W(\varphi(\lambda \cdot))]\right\|_{W\left(\mathcal{F} L^{p}, L^{q}\right)}
\end{aligned}
$$

for any $\chi \in C_{c}^{\infty}(\mathbb{R})$ and $n \in \mathbb{N}_{+}$. Choosing $\chi$ supported in the interval $[-1 / 4,1 / 4]$ and $\chi \equiv 1$ in the interval $[-1 / 8,1 / 8]$ (the latter condition will be used later), we write

$$
\chi\left(\zeta_{1} \zeta_{2}\right)=\chi\left(\zeta_{1} \zeta_{2}\right) \Theta^{n}\left(\zeta_{1}, \zeta_{2}\right) \Theta^{-n}\left(\zeta_{1}, \zeta_{2}\right) \tilde{\chi}\left(\zeta_{1} \zeta_{2}\right)
$$

with $\tilde{\chi} \in C_{c}^{\infty}(\mathbb{R})$ supported in $[-1 / 2,1 / 2]$ and $\tilde{\chi}=1$ on $[-1 / 4,1 / 4]$, therefore on the support of $\chi$. Since by Lemma 5.2 the function $\Theta^{-n}\left(\zeta_{1}, \zeta_{2}\right) \tilde{\chi}\left(\zeta_{1} \zeta_{2}\right)$ belongs to $W\left(\mathcal{F} L^{1}, L^{\infty}\right)$, by the product property the last expression can be estimated from below as

$$
\gtrsim\left\|\chi\left(\zeta_{1} \zeta_{2}\right) \mathcal{F}_{\sigma}[W(\varphi(\lambda \cdot))]\right\|_{W\left(\mathcal{F} L^{p}, L^{q}\right)}
$$

We are ended up with the same object already estimated in the proof of Theorem 1.4 in [12], were it was shown that

$$
\left\|\chi\left(\zeta_{1} \zeta_{2}\right) \mathcal{F}_{\sigma}[W(\varphi(\lambda \cdot))]\right\|_{W\left(\mathcal{F} L^{p}, L^{q}\right)} \gtrsim \lambda^{-2 d+d / p+d / q} \quad \text { as } \lambda \rightarrow+\infty
$$

Comparing (5.5) with (5.4) we obtain the desired conclusion. 


\section{Pseudodifferential Calculus}

The Weyl quantization was introduced by Weyl in [48] and is the $n=0$ case of the Born-Jordan quantization of order $n$ in (1.14):

$$
a \in \mathcal{S}^{\prime}\left(\mathbb{R}^{2 d}\right) \mapsto \widehat{A}_{\mathrm{W}}=\mathrm{Op}_{\mathrm{W}}(a)=\left(\frac{1}{2 \pi \hbar}\right)^{d} \int_{\mathbb{R}^{2 d}} \mathcal{F}_{\sigma} a(z) \widehat{T}(z) d z
$$

Comparing with (1.14), we infer the symbol relation

$$
\mathcal{F}_{\sigma} a_{B J, n} \Theta^{n}=\mathcal{F}_{\sigma} a_{W}
$$

(observe that $a_{B J, n}$ denotes the symbol of $\widehat{A}_{\mathrm{BJ}, n}$ whereas $a_{W}$ is the Weyl symbol) that is

$$
a_{B J, n} * \mathcal{F}_{\sigma}\left(\Theta^{n}\right)=a_{W} .
$$

Using the weak definition for Weyl operators via the Wigner distribution

$$
\left\langle O p_{W}(a) f, g\right\rangle=\langle a, W(g, f)\rangle, \quad a \in \mathcal{S}^{\prime}\left(\mathbb{R}^{2 d}\right), f, g \in \mathcal{S}\left(\mathbb{R}^{d}\right)
$$

and the convolution property (whenever is well-defined)

$$
\langle F * G, H\rangle=\langle F, H * G\rangle
$$

we can also define, for $n \in \mathbb{N}$, the $n$-th Born-Jordan pseudodifferential operator with symbol $a \in \mathcal{S}^{\prime}\left(\mathbb{R}^{d}\right)$ by

$$
\left\langle O p_{B J, n}(a) f, g\right\rangle=\left\langle a, Q^{n}(g, f)\right\rangle, \quad f, g \in \mathcal{S}\left(\mathbb{R}^{d}\right) .
$$

(Observe that $n=1$ is the standard BJ operator, whereas $n=0$ gives the Weyl one).

We aim at studying continuity properties of such operators and of the related distributions on modulation spaces.

First, we analyze the quadratic representations $Q^{n}$.

Theorem 6.1. Assume $s \geq 0, p_{1}, q_{1}, p, q \in[1, \infty]$ such that

$$
2 \min \left\{\frac{1}{p_{1}}, \frac{1}{q_{1}}\right\} \geq \frac{1}{p}+\frac{1}{q} .
$$

If $f \in M_{v_{s}}^{p_{1}, q_{1}}\left(\mathbb{R}^{d}\right)$ the Cohen distribution $Q^{n} f, n \in \mathbb{N}_{+}$, is in $M_{1 \otimes v_{s}}^{p, q}\left(\mathbb{R}^{2 d}\right)$, with

$$
\left\|Q^{n} f\right\|_{M_{1 \otimes v_{s}}^{p, q}\left(\mathbb{R}^{2 d}\right)} \lesssim\left\|\Theta^{n}\right\|_{W\left(\mathcal{F} L^{1}, L^{\infty}\right)\left(\mathbb{R}^{2 d}\right)}\|f\|_{M_{v_{s}}^{p_{1}, q_{1}}\left(\mathbb{R}^{d}\right)}^{2} .
$$

Proof. In [13, Theorem 1.2] two of us proved that, if the Cohen kernel $\theta$, defined in (1.2), is in $M^{1, \infty}\left(\mathbb{R}^{2 d}\right)$, then the related Cohen distribution $Q f$ satisfies

$$
\left\|Q^{n} f\right\|_{M_{1 \otimes v_{s}}^{p, q}\left(\mathbb{R}^{2 d}\right)} \lesssim\|\theta\|_{M^{1, \infty}\left(\mathbb{R}^{2 d}\right)}\|f\|_{M_{v_{s}}^{p_{1}, q_{1}}\left(\mathbb{R}^{d}\right)}^{2}
$$

where the indices $p_{1}, q_{1}, p, q \in[1, \infty]$ are related by condition $(6.3)$. 
By Proposition 4.2, the function $\Theta^{n}$ is in $W\left(\mathcal{F} L^{1}, L^{\infty}\right)$, so that the BJ kernel $\mathcal{F}_{\sigma}\left(\Theta^{n}\right)$ is in $M^{1, \infty}\left(\mathbb{R}^{2 d}\right)$ with $\left\|\mathcal{F}_{\sigma}\left(\Theta^{n}\right)\right\|_{M^{1, \infty}} \asymp\left\|\Theta^{n}\right\|_{W\left(\mathcal{F} L^{1}, L^{\infty}\right)}$ and the thesis follows.

We write $q^{\prime}$ for the conjugate exponent of $q \in[1, \infty]$; it is defined by $1 / q+1 / q^{\prime}=$ 1. The $n$-th Born-Jordan operator enjoys the same continuity properties as for the $n=1$ case, proved in [11, Theorem 1.1]. Indeed, we can state:

Theorem 6.2. Consider $1 \leq p, q, r_{1}, r_{2} \leq \infty$, such that

$$
p \leq q^{\prime}
$$

and

$$
q \leq \min \left\{r_{1}, r_{2}, r_{1}^{\prime}, r_{2}^{\prime}\right\}
$$

Then the Born-Jordan operator $O p_{B J, n}(a)$, from $\mathcal{S}\left(\mathbb{R}^{d}\right)$ to $\mathcal{S}^{\prime}\left(\mathbb{R}^{d}\right)$, having symbol $a \in M^{p, q}\left(\mathbb{R}^{2 d}\right)$, extends uniquely to a bounded operator on $\mathcal{M}^{r_{1}, r_{2}}\left(\mathbb{R}^{d}\right)$, with the estimate

$$
\left\|O p_{B J, n}(a) f\right\|_{\mathcal{M}^{r_{1}, r_{2}}} \lesssim\|a\|_{M^{p, q}}\|f\|_{\mathcal{M}^{r_{1}, r_{2}}}, \quad f \in \mathcal{M}^{r_{1}, r_{2}} .
$$

Vice versa, if this conclusion holds true, the constraints (6.5) is satisfied and it must hold

$$
\max \left\{\frac{1}{r_{1}}, \frac{1}{r_{2}}, \frac{1}{r_{1}^{\prime}}, \frac{1}{r_{2}^{\prime}}\right\} \leq \frac{1}{q}+\frac{1}{p}
$$

that is (6.6) for $p=\infty$.

Proof. The sufficient conditions are proved by induction. The result holds true for $n=1$ by Theorem [11, Theorem 1.1]. Assume now that the result is true for a certain $n \in \mathbb{N}_{+}$and observe, by definition (1.14), that

$$
O p_{B J, n+1}(a)=O p_{B J, n}(b), \quad \text { with } \quad a=b * \mathcal{F}_{\sigma} \Theta .
$$

The thesis follows by the convolution relation $M^{p, q}\left(\mathbb{R}^{2 d}\right) * M^{1, \infty}\left(\mathbb{R}^{2 d}\right) \hookrightarrow M^{p, q}\left(\mathbb{R}^{2 d}\right)$.

The necessary conditions are obtained arguing exactly as for the case $n=1$, for details we refer to the proof of Theorem 1.1 in [11].

\section{TECHNICAL NOTES}

The figures in the introduction were produced using LTFAT (The Large TimeFrequency Analysis Toolbox), cf. [43] as well as the Time-Frequency Toolbox (TFTB), distributed under the terms of the GNU Public Licence:

$$
\text { http://tftb.nongnu.org/ }
$$

The bat sonar signal in Figure 3 was recorded as a .mat file in the latter toolbox. 


\section{ACKNowledgments}

The authors would like to thank Professor Jean-Pierre Gazeau, for inspiring this work during the wonderful environment of the conference Quantum Harmonic Analysis and Symplectic Geometry, April 21-24, 2018, Strobl, AUSTRIA. Maurice de Gosson has been financed by the grant P27773 of the Austrian research Foundation FWF. Monika Dörfler has been supported by the Vienna Science and Technology Fund (WWTF) through project MA14-018.

\section{REFERENCES}

[1] P. Boggiatto, E. Carypis, A. Oliaro, Windowed-Wigner representations in the Cohen class and uncertainty principles, J. Geom. Anal., 23(4):1753-1779, 2013.

[2] P. Boggiatto, G. De Donno, A. Oliaro, Time-frequency representations of Wigner type and pseudo-differential operators, Trans. Amer. Math. Soc., 362(9): 4955-4981, 2010.

[3] P. Boggiatto, A. Oliaro, P. Wahlberg, The Wave Front Set of the Wigner Distribution and Instantaneous Frequency, J. Fourier Anal. Appl., 18(2) (2012), 410-438.

[4] O. Christensen, An Introduction to Frames and Riesz Bases, Birkhäuser, Basel, 2016.

[5] H. Choi, W.J. Williams, Improved time-frequency representation of multicomponent signals using exponential kernels, IEEE Trans. Acoust., Speech, Signal Process., 37(6) (1989), 862871.

[6] L. Cohen, Generalized phase-space distribution functions, J. Math. Phys., 7 (1966), 781-786.

[7] L. Cohen, Time Frequency Analysis: Theory and Applications, Prentice Hall, 1995.

[8] L. Cohen, The Weyl operator and its generalization, Springer Science \& Business Media, 2012.

[9] E. Cordero, F. Nicola, Metaplectic representation on Wiener amalgam spaces and applications to the Schrödinger equation, J. Funct. Anal., 254 (2008), 506-534.

[10] E. Cordero, M. de Gosson, F. Nicola, On the invertibility of Born-Jordan quantization, J. Math. Pures Appl., 105 (2016), 537-557.

[11] E. Cordero, M. de Gosson, F. Nicola, Time-frequency Analysis of Born-Jordan Pseudodifferential Operators. J. Funct. Anal., 272(2):577-598, 2017. DOI:10.1016/j.jfa.2016.10.004

[12] E. Cordero, M. de Gosson and F. Nicola. On the reduction of the interferences in the Born-Jordan distribution. Appl. Comput. Harmon. Anal., 44(2):230-245, 2018. DOI:10.1016/j.acha.2016.04.007

[13] E. Cordero and F. Nicola, Sharp Integral Bounds for Wigner Distribution, International Mathematics Research Notices, 2016(00): 1-29, 2016

[14] N. G. de Bruijn, A theory of generalized functions, with applications to Wigner distribution and Weyl correspondence, Njeuw Archief voor Wiskunde, (21):205-280, 1973.

[15] H.B. Domingo and E.A. Galapon, Generalized Weyl transform for operator ordering: polynomial functions in phase space. J. Math. Phys. 56(2), 022104, 2015.

[16] F. Faà di Bruno, Sullo Sviluppo delle Funzioni, Annali di Scienze Matematiche e Fisiche, (6):479-480, 1855.

[17] H. G. Feichtinger, On a new Segal algebra, Monatshefte Math., 92(4): 269-289, 1981.

[18] H. G. Feichtinger, Modulation spaces on locally compact abelian groups, Technical Report, University Vienna, 1983, and also in "Wavelets and Their Applications", M. Krishna, R. Radha, S. Thangavelu, editors, Allied Publishers, 99-140, 2003. 
[19] H. G. Feichtinger, Banach convolution algebras of Wiener's type, In Proc. Conf. "Function, Series, Operators", Budapest August 198, Colloq. Math. Soc. János Bolyai, 35, 509-524, North-Holland, Amsterdam, 1983.

[20] H. G. Feichtinger, Banach spaces of distributions of Wiener's type and interpolation. In "Proc. Conf. Functional Analysis and Approximation, Oberwolfach August 1980", Internat. Ser. Numer. Math., 69:153-165. Birkhäuser, Boston, 1981.

[21] H. G. Feichtinger, Generalized amalgams, with applications to Fourier transform, Canad. J. Math., 42(3): 395-409, 1990.

[22] H. G. Feichtinger, G. Zimmermann, A Banach space of test functions for Gabor analysis. In "Gabor Analysis and Algorithms: Theory and Applications", H.G. Feichtinger and T. Strohmer (eds.), Applied and Numerical Harmonic Analysis, Birkhäuser, Basel 1998, 123170 .

[23] G. B. Folland, Harmonic Analysis in Phase Space, Princeton Univ. Press, Princeton, NJ, 1989.

[24] L. Galleani, L. Lo Presti, Application of the Wigner distribution to nonlinear systems, Journal of Modern Optics, 49(3/4):571-579, 2002.

[25] M. de Gosson, Symplectic methods in Harmonic Analysis and in Mathematical Physics, Birkhäuser, 2011.

[26] M. de Gosson, Symplectic covariance properties for Shubin and Born-Jordan pseudodifferential operators, Trans. Amer. Math. Soc., 365(6):3287-3307, 2013.

[27] M. de Gosson, Born-Jordan Quantization: Theory and Applications. Vol. 182, Springer, 2016.

[28] M. de Gosson. The Wigner Transform, World Scientific Pub Co, 2017.

[29] M. de Gosson, F. Luef, Preferred quantization rules: Born-Jordan vs. Weyl; applications to phase space quantization, J. Pseudo-Differ. Oper. Appl., 2(1): 115-139, 2011.

[30] K. Gröchenig, Foundation of Time-Frequency Analysis, Birkhäuser, Boston MA, 2001.

[31] K. Gröchenig. Time-Frequency Analysis of Sjöstrand's Class. Rev. Mat. Iberoamericana, $22(2): 703-724,2006$.

[32] F. Hlawatsch, P. Flandrin, The interference structure of the Wigner distribution and related time-frequency signal representations, W. Mecklenbräuker, F. Hlawatsch, Eds., The Wigner Distribution - Theory and Applications in Signal Processing, 59-133, Elsevier, Amsterdam, The Netherlands, 1997.

[33] F. Hlawatsch, F. Auger, Time-Frequency Analysis, Wiley, 2008.

[34] L. Hörmander, Lectures on nonlinear hyperbolic differential equations, Springer, 1997.

[35] A. J. E. M. Janssen, On the locus and spread of pseudodensity functions in the time-frequency plane, Philips J. Res., 37(3):79-110, 1982.

[36] J. Jeong, W.J. Williams, Kernel design for reduced interference distributions, IEEE Trans. Signal Process., 40(2):402-412, 1992.

[37] G. Kutyniok, D. Labate, Resolution of the wavefront set using continuous shearlets, Trans. Amer. Math. Soc., 361:2719-2754, 2009.

[38] P. Loughlin, J. Pitton, L. Atlas, New properties to alleviate interference in time-frequency representations, Proc. IEEE Intl. Conf. Acous., Speech and Sig. Process.'91, pp. 3205-3208, 1991.

[39] P. Loughlin, J. Pitton, L. Atlas, Bilinear time-frequency representations: New insights and properties, IEEE Trans. Sig. Process., 41(2):750-767, 1993.

[40] S. Pilipović, N. Teofanov, J. Toft, Wave front sets in Fourier Lebesgue spaces, Rend. Sem. Mat. Univ. Pol. Torino, 66(4):299-319, 2008. 
[41] S. Pilipovic, N. Teofanov, J. Toft, Micro-local analysis in Fourier-Lebesgue and modulation spaces. Part II, J. Pseudo-Differ. Oper. Appl., (1) 341-376, 2010.

[42] S. Pilipovic, N. Teofanov, J. Toft, Micro-Local Analysis in Fourier-Lebesgue and modulation Spaces. Part I, J. Fourier Anal. Appl., 17:374-407, 2011.

[43] Z.Prusa, P. L. Sndergaard, N.Holighaus, C. Wiesmeyr, P. Balazs, The Large Time-Frequency Analysis Toolbox 2.0. Sound, Music, and Motion, Lecture Notes in Computer Science 2014, pp 419-442

[44] H. Reiter, Metaplectic Groups and Segal Algebras, Lecture Notes in Mathematics 1382, Springer-Verlag, Berlin, 1989.

[45] M. Sugimoto, N. Tomita. The dilation property of modulation spaces and their inclusion relation with Besov spaces, J. Funct. Anal., 248(1) (2007), 79-106.

[46] J. Toft, Continuity properties for modulation spaces, with applications to pseudo-differential calculus. I, J. Funct. Anal., 207(2): 399-429, 2004.

[47] V. Turunen, Born-Jordan time-frequency analysis, Conference slides, Turin, December 10th, 2014.

[48] H. Weyl, Quantenmechanik und Gruppentheories. Zeitschrift fur Physik, 46:1-46, 1927.

[49] Y. Zhao, L.E. Atlas, R.J. Marks, The use of cone-shaped kernels for generalized timefrequency representations of nonstationary signals, IEEE Trans. Acoust., Speech, Signal Process., 38 (7):1084-1091, 1990.

Dipartimento di Matematica, Università di Torino, Dipartimento di Matematica, via Carlo Alberto 10, 10123 Torino, Italy

E-mail address: elena.cordero@unito.it

University of Vienna, Faculty of Mathematics, Oskar-Morgenstern-Platz 1 A1090 Wien, Austria

E-mail address: maurice.de.gosson@univie.ac.at

University of Vienna, Faculty of Mathematics, Oskar-Morgenstern-Platz 1 A1090 Wien, Austria

E-mail address: monika.doerfler@univie.ac.at

Dipartimento di Scienze Matematiche, Politecnico di Torino, corso Duca degli Abruzzi 24, 10129 Torino, Italy

E-mail address: fabio.nicola@polito.it 